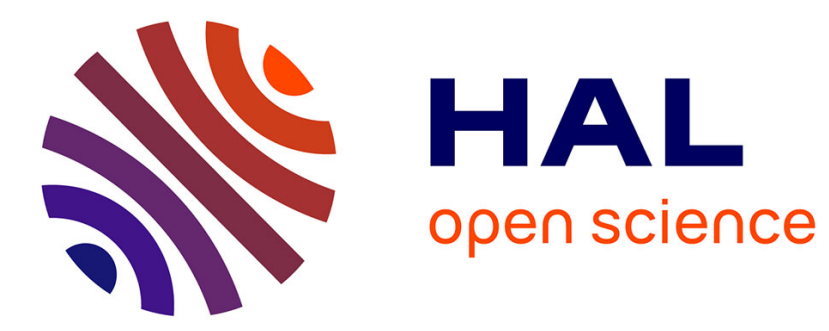

\title{
Les défauts de couleur du tissu adipeux sous-cutané des carcasses d'ovins
}

\author{
Sophie Prache, B. Aurousseau, M. Theriez, M. Renerre
}

\section{To cite this version:}

Sophie Prache, B. Aurousseau, M. Theriez, M. Renerre. Les défauts de couleur du tissu adipeux sous-cutané des carcasses d'ovins. Productions Animales, 1990, 3 (4), pp.275-285. hal-00895911

\section{HAL Id: hal-00895911 \\ https://hal.science/hal-00895911}

Submitted on 1 Jan 1990

HAL is a multi-disciplinary open access archive for the deposit and dissemination of scientific research documents, whether they are published or not. The documents may come from teaching and research institutions in France or abroad, or from public or private research centers.
L'archive ouverte pluridisciplinaire HAL, est destinée au dépôt et à la diffusion de documents scientifiques de niveau recherche, publiés ou non, émanant des établissements d'enseignement et de recherche français ou étrangers, des laboratoires publics ou privés. 
INRA Prod. Anim. 1990, 3 (4), $275-285$
Sophie PRACHE (1), B. AUROUSSEAU (2) M. THERIEZ (1), M. RENERRE (3)

\section{INRA}

(1) Laboratoire de la Lactation

et de l'Elevage des Ruminants

(2) Laboratoire de la Croissance

et des Métabolismes des Herbivoires

(3) Station de Recherches sur la Viande

Theix 63122 Saint-Genès-Champanelle
Les défauts de couleur du tissu adipeux sous-cutané des carcasses d'ovins

\section{Une enquête réalisée en 1989 par quelques groupements de producteurs}

a montré que 20 à $25 \%$ des carcasses d'agneaux présentent des défauts de couleur du tissu adipeux de couverture (normalement blanc). Les carcasses excessivement pigmentées en jaune sont généralement saisies à l'abattoir car ce défaut peut avoir une origine pathologique. La coloration brun-rouge conduit à une pénalisation commerciale ; elle s'accompagne le plus souvent d'un défaut de fermeté qui rend la découpe difficile et nuit à la bonne présentation des morceaux. Elle pourrait aussi être associée à des arômes et des saveurs désagréables lors de la cuisson.

La qualité des carcasses d'ovins dépend de nombreux éléments dont le poids, la conformation, les caractéristiques du tissu adipeux, la couleur de la viande, et éventuellement l'odeur. Les caractéristiques du tissu adipeux (quantité, fermeté, couleur) ont des répercussions de plus en plus importantes sur la valeur marchande

\section{Résumé}

Les caractéristiques de couleur et de fermeté de 27 échantillons de tissu adipeux récoltés en abattoir ( 4 blancs, 4 jaunes, 19 brun-rouge) ont été décrites par différentes méthodes utilisables en abattoir ou en laboratoire (en particulier, la spectrocolorimétrie). Pour certains de ces échantillons, des informations concernant les conditions d'élevage des animaux ont pu être collectées.

Les tissus adipeux blancs « de référence " étaient fermes et correspondaient à des agneaux produits essentiellement à l'herbe. Nous y avons trouvé une faible quantité de pigments héminiques, probablement de l'oxyhémoglobine résiduelle après saignée.

Les tissus adipeux jaunes étaient colorés par des pigments héminiques, et par des pigments caroténoïdes et/ou de la bilirubine et présentaient des caractéristiques de fermeté assez proches de celles des tissus adipeux blancs. Les origines possibles de ces défauts de coloration sont présentées.

Les tissus adipeux brun-rouge provenaient généralement d'animaux ayant reçu des rations riches en concentré. Ces tissus présentaient des défauts de fermeté dus à une diminution de leur teneur en lipides et à une modification de leur composition en acides gras. Le défaut de coloration a pu être attribué 1) à un effet du degré de fermeté du tissu adipeux sur la réflexion de la lumière, 2) à l'accumulation excessive de pigments héminiques. Celle-ci résulte de la fragilisation de la membrane des érythrocytes du fait des caractéristiques de composition en acides gras des phospholipides, 3) aux produits de la peroxydation des acides gras insaturés, elle même stimulée par ces pigments héminiques. d'une carcasse. Les facteurs de variation de la quantité et de la fermeté sont bien connus et ont été revus par Thériez et al (1976) et Aurousseau (1986). En revanche, les causes de l'apparition de colorations anormales du tissu adipeux le sont moins bien.

Le tissu adipeux de couverture des carcasses d'ovins est normalement blanc. Le développement de couleurs inhabituelles (jaune, brun, rouge, bis) peut aboutir, soit à la saisie de la carcasse dans les cas les plus graves, soit à une dépréciation commerciale compte tenu du rejet probable par les consommateurs. Une première année d'observation en abattoir réalisée en 1989 par différents groupements de producteurs (tableau 1) a permis de chiffrer l'importance du problème : de $20 \%$ à $25 \%$ des carcasses présentent des défauts de couleur du tissu adipeux importants à très importants.

On distingue deux types de coloration du tissu adipeux de couverture, jaune et brunrouge.

- La coloration jaune (photo 1) : elle apparaît dès l'abattage, et doit correspondre, d'après la littérature à l'accumulation excessive de pigments caroténoïdes et/ou de bilirubine.

Les ovins n'accumulent normalement pas les caroténoïdes dans leur tissu adipeux, même lorsque leur alimentation en comporte (Rozier 1979). Cependant, l'existence de carcasses d'ovins excessivement pigmentées en jaune par des caroténoïdes est évoquée depuis de nombreuses années dans divers pays. Le problème 
Tableau 1. Premiers résultats des enquêtes réalisées par des organismes de développement dans différentes régions, concernant les défauts de couleur du tissu adipeux de couverture de carcasses d'agneaux.

\begin{tabular}{|c|c|c|c|c|}
\hline \multirow{2}{*}{$\begin{array}{c}\text { Organisme } \\
\text { de développement }\end{array}$} & \multirow{2}{*}{$\begin{array}{c}\text { Nombre } \\
\text { de carcasses } \\
\text { observées }\end{array}$} & \multicolumn{3}{|c|}{ Couleur du tissu adipeux } \\
\hline & & Correcte & Intermédiaire & $\begin{array}{c}\text { Mauvaise } \\
\text { à très mauvaise }\end{array}$ \\
\hline GIE ovin Centre Ouest (1) & 8560 & $70 \%$ & $10 \%$ & $20 \%$ \\
\hline COPA Haute Auvergne (2) & 12530 & $23 \%$ & $53 \%$ & $24 \%$ \\
\hline GEBRO (Aveyron) (2) & 1140 & $22,5 \%$ & $52,5 \%$ & $25 \%$ \\
\hline
\end{tabular}

(1) Zone de production d'agneaux d'herbe essentiellement.

(2) Zone de production d'agneaux de bergerie essentiellement.

concernait une carcasse sur 200 en Norvège en 1983 (Baker et al 1985), 1 carcasse sur 500 en Irlande et en Nouvelle Zélande en 1980 (Kirton 1980), avec des fréquences de 5 à $10 \%$ occasionnellement constatées dans certaines exploitations (Kirton et al 1975, Kirton 1980). Les auteurs s'accordent pour dire que ces carcasses ne présentent pas de lésions particulières ni d'altération de la qualité de leur viande, et qu'elles sont parfaitement saines et consommables par l'homme (Rozier et al 1979, Kirton 1980, Baker et al 1985). A l'inverse, l'accumulation excessive de bilirubine dans les tissus peut avoir des origines diverses qui, toutes, sont pathologiques. Cependant, la difficulté de distinction des deux types de pigments lors de l'inspection en abattoir conduit généralement à la saisie de toutes les carcasses excessivement jaunes.

- La coloration brun-rouge (photos 1, 2, 3), qui s'accompagne le plus souvent d'un défaut de fermeté du tissu adipeux (photo 4) et semble se développer au cours du temps de ressuyage, ne provoque généralement pas la saisie de la carcasse, mais plutôt une pénalisation commerciale. Elle pourrait être associée à des risques d'arômes et de saveurs désagréables lors de la cuisson (Smith et Carpenter 1970). Le développement d'une coloration brune sur du gras de couverture présentant également des défauts de fermeté a déjà été constaté par L'Estrange et Mulvihill (1975) au cours du ressuyage de carcasses d'agneaux de bergerie, mais sans recherche de l'origine du problème. Ce défaut de coloration du tissu adipeux a également été signalé sur des porcs, des volailles, des rats, et même chez l'homme.

Des échantillons de tissu adipeux blancs, jaunes et brun-rouge ont donc été récoltés en abattoir, afin de faire une analyse détaillée de leurs caractéristiques de coloration et de fermeté en développant en particulier des méthodes objectives d'appréciation de la couleur et de détection des pigments responsables. Nous avons également fait le point sur les connaissances actuelles quant aux causes d'apparition de ces défauts de coloration.

\section{CONDITIONS EXPERIMENTALES}

\section{1 / Origine des échantillons de tissu adipeux analysés}

Compte tenu de la grande variabilité des colorations observées et/ou décrites par les techniciens des groupements, nous avons comparé 23 carcasses présentant des défauts de couleur à un groupe de 4 carcasses de référence (gras blanc et dur). Parmi ces 23 carcasses, 4 ont été classées en jaune et les 19 autres en brun-rouge avec, ici encore, une variabilité qui sera analysée dans la suite de l'article.

Ces carcasses provenaient de groupements de producteurs situés dans les régions PoitouCharentes, Limousin, Auvergne et Midi-Pyrénées (Aveyron), d'une Chambre d'Agriculture (Alsace), et de l'INRA de Theix. Les échantillons de tissu adipeux, prélevés à la base de la queue le lendemain de l'abattage après ressuyage en chambre froide, ont été conservés au froid et à l'abri de la lumière dans du papier aluminium jusqu'à l'analyse.

Des informations concernant les conditions d'élevage des animaux ont pu être obtenues chez les producteurs, pour 16 d'entre eux ( 3 blancs, 1 jaune, 12 brun-rouge).

\section{2 / Mesures réalisées et méthodes utilisées}

\section{1 / Couleur du tissu adipeux}

Nous avons apprécié la couleur des échantillons par comparaison avec des étalons colorés (Food colorimetry : theory and application, 1975). Les gras jaunes correspondaient aux étalons Munsell 5Y9/2, $5 \mathrm{Y9} / 4$ et $5 \mathrm{Y9} / 6$; les gras brun-rouge aux étalons 5 YR $8 / 6,5$ YR $8 / 8$, 7,5 YR8/6, 7.5YR7/8, 7.5YR8/8, 10 YR9/4, $10 Y R 8 / 6$ et $10 Y R 8 / 8$. Il n'a pas été possible de 
trouver d'étalons correspondant aux gras blancs.

Le spectre de réflectance de ces échantillons a été mesuré dans le visible (400-750 nm), avec un spectrocolorimètre Beckman DB-GT (illuminant $\mathrm{C}$ ) et les résultats ont été exprimés dans les coordonnées du système CIELAB (1976): luminosité $\left(\mathrm{L}^{\star}\right)$, indice de rouge $\left(\mathrm{a}^{\star}\right)$, indice de jaune $\left(b^{*}\right)$ (Renerre 1988). Les échantillons, stockés à $+4^{\circ} \mathrm{C}$, ont été analysés à température ambiante 4 à 8 heures après leur prélèvement, sauf les 2 échantillons en provenance d'Alsace (échantillons jaunes $n^{\circ} 1$ et 3 , stockés à - $18^{\circ} \mathrm{C}$ pendant 1 mois).

\section{2 / Nature et concentration des pigments présents dans le tissu adipeux}

\section{a / Bilirubine et pigments caroténoïdes}

Les concentrations du tissu adipeux en bilirubine et pigments caroténoïdes ont été analysées sur 15 des 27 échantillons prélevés ( 4 blancs, 4 jaunes et 7 brun-rouge). Les deux types de pigments ont d'abord été séparés après extraction du tissu adipeux (Aurousseau et al, non publié), puis la concentration de chacun d'entre eux a été estimée par spectrophotométrie (spectrophotomètre KONTRON UVIKON 820) après calibration avec la bilirubine d'une part, et la zéaxanthine d'autre part. Ce dosage a été réalisé dans un délai d'un mois après le prélèvement.

\section{b / Pigments héminiques}

Un premier examen de la nature des pigments héminiques présents dans un échantillon de tissu adipeux brun-rouge a été réalisé après extraction dans un tampon. Les pigments hydrosolubles ont été séparés par chromatographie d'exclusion sur colonne et les spectres d'absorption correspondants ont été enregistrés dans le visible à l'aide d'un spectrocolorimètre.

\section{3 / Fermeté du tissu adipeux}

Elle a été estimée, au moment du prélèvement en chambre froide par palpation du gras dorsal ( 4 classes ont été définies : ferme, moyen, mou, huileux). En laboratoire, la teneur $\mathrm{du}$ tissu adipeux en lipides et sa composition en acides gras ont été mesurées sur les 15 échantillons dont la concentration en pigments a été analysée. Les méthodes utilisées ont été respectivement la pesée après extraction et la chromatographie en phase gazeuse (Bauchart et Aurousseau 1981). Les analyses ont été réalisées dans un délai d'un mois après le prélèvement.

\section{RESULTATS ET DISCUSSIONS}

\section{1 / Conditions d'élevage des animaux}

Nous disposons de précisions quant aux conditions d'élevage des animaux pour 16 échantillons ( 3 blancs, 1 jaune, 12 brunrouge) :

- pour les échantillons blancs, il s'agit d'agneaux élevés exclusivement à l'herbe (2 agneaux) ou complémentés à volonté à l'herbe avec du concentré puis fini pendant 20 jours en bergerie (1 agneau),

- pour l'échantillon jaune, il s'agit d'un agneau d'herbe fini pendant 45 jours sur une luzernière avec $400 \mathrm{~g} / \mathrm{j}$ de concentré avant l'abattage à 195 jours,

pour les échantillons brun-rouge, le mode d'élevage est très variable: élevage intégral en bergerie ( 3 agneaux), élevage à l'herbe avec distribution à volonté de concentré et de foin $(2$ agneaux), ou élevage à l'herbe et finition soit en bergerie pendant 20 à 45 jours ( 5 agneaux) soit sur colza fourrager pendant 45 jours ( 2 agneaux). Onze de ces 12 animaux à gras brunrouge étaient des mâles.

\section{2 / Caractérisation des différentes couleurs}

\section{1 / Spectres de réflectance}

a / Les types de spectre : cinq spectres caractéristiques des différents types de gras sont reportés sur la figure 1: gras blancs (1), gras jaunes (2) et gras brun-rouge (2). Ces spectres présentent en commun des maxima d'absorption qui se situent à des longueurs d'onde très voisines, mais se différencient par contre par la présence de maxima spécifiques et par la proportion de lumière réfléchie.

Figure 1. Spectres de réflectance moyens des échantillons de tissu adipeux.

\section{Proportion de}

lumière réfléchie (\%)

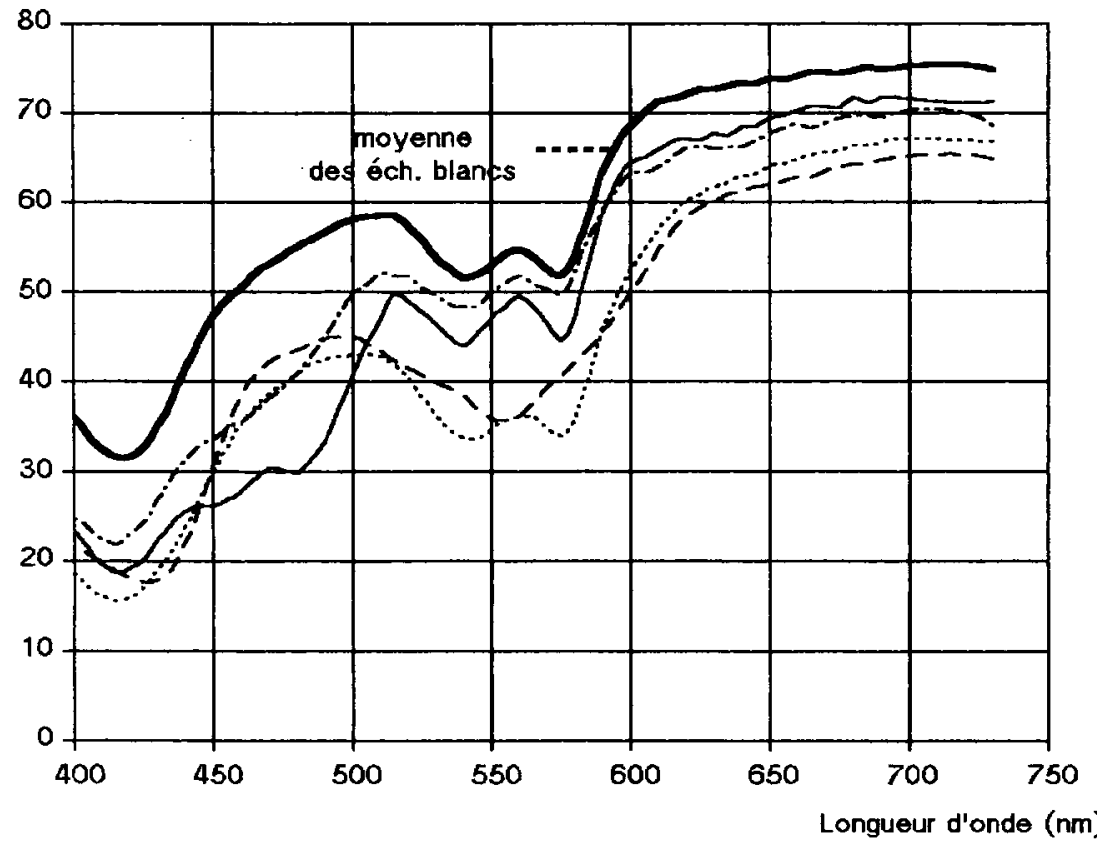

tissus adipeux blancs / _ tissus adipeux jaunes $n^{\prime \prime} 1$ et $n^{\prime \prime} 2$ - - tissus adipeux jaunes $n^{\circ} 3$ et $n^{\circ} 4 /--$ tissus adipeux brun-rouge de type $I / \ldots$ tissus adipeux brun-rouge de type II. 


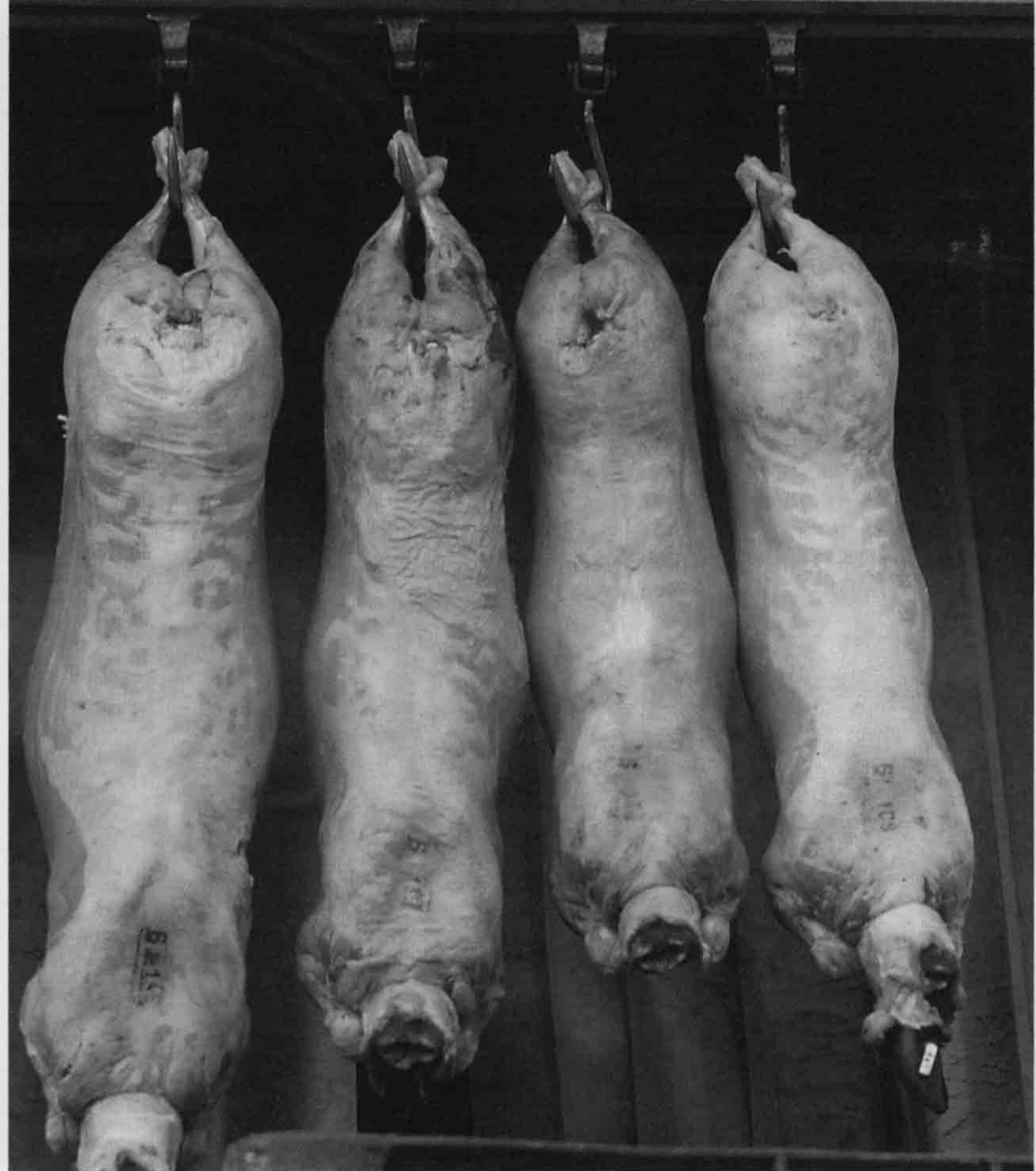

De gauche à droite : une carcasse excessivement pigmentée en jaune par des caroténoïdes (saisie), deux carcasses présentant un gras brun-rouge, une carcasse correcte (gras blanc).

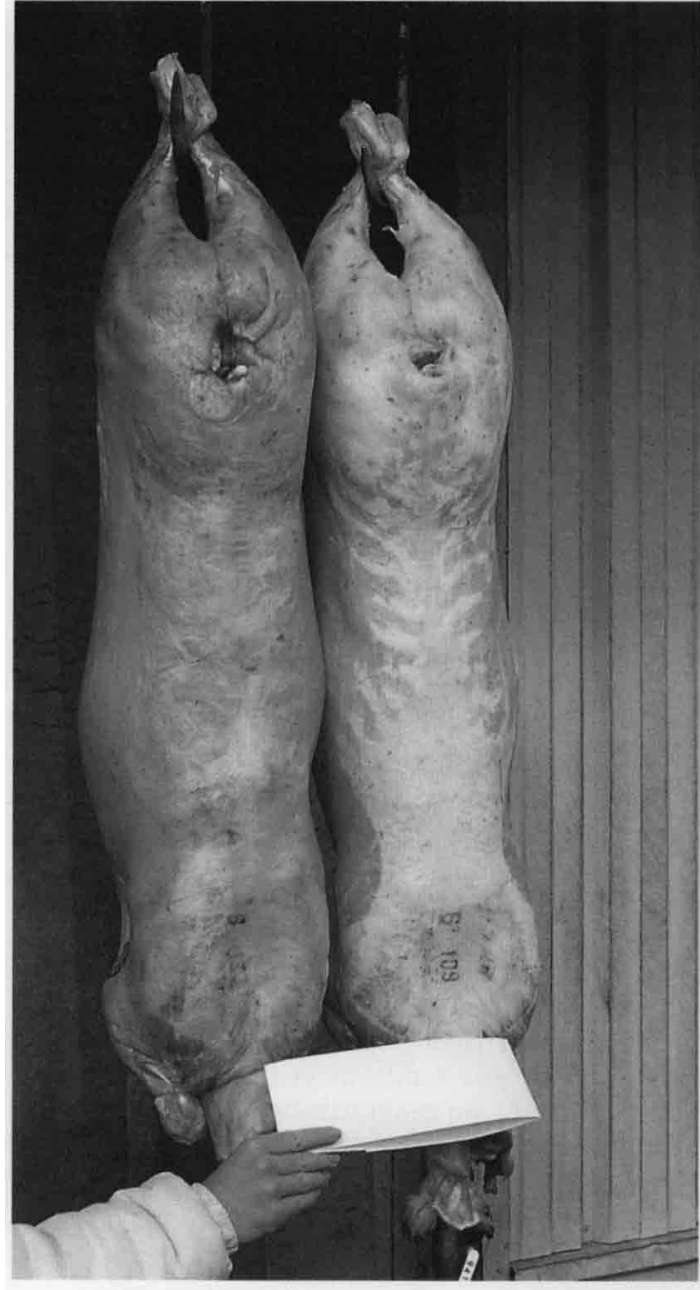

La carcasse de droite a une couleur correcte, celle de gauche présente un gras brun-rouge.

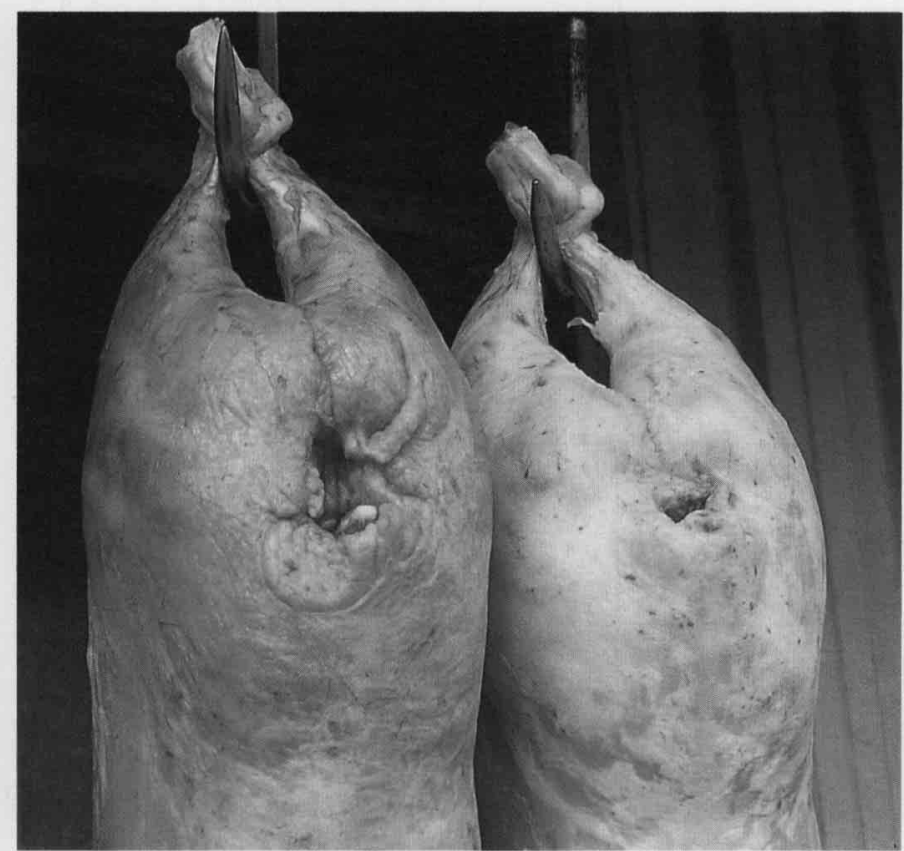

Défaut de fermeté du gras de couverture: le gras est huileux, même après ressuyage.

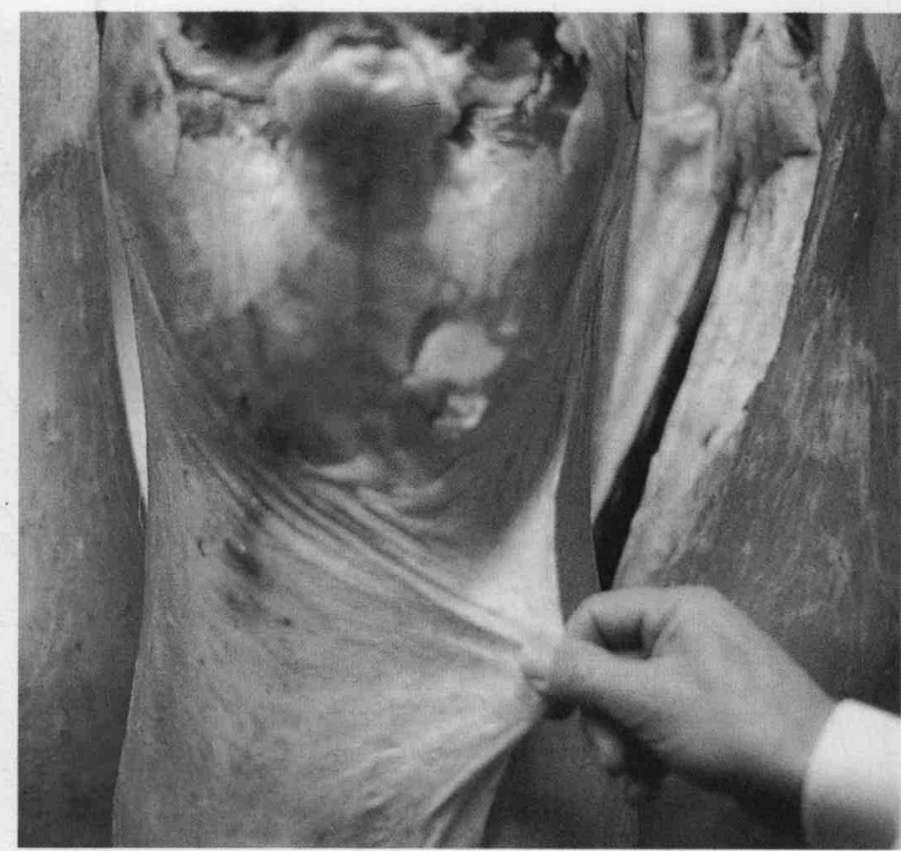

Gigots à gras de couverture brun-rouge (à gauche), et blano (à droite). 
Tableau 2. Caractéristiques colorimétriques, concentration en caroténoïdes et en bilirubine de tissus adipeux sous-cutanés prélevés sur des carcasses d'agneaux de boucherie de couleur blanche, jaune ou brun-rouge (moyenne et écart type).

\begin{tabular}{|c|c|c|c|c|c|}
\hline Couleur & Blanc & Jaune & \multicolumn{3}{|c|}{ Brun-rouge } \\
\hline Type & & & I & Intermédiaire & II \\
\hline $\begin{array}{l}\text { Nombre d'échantillons } \\
\text { Indices CIELAB } \\
\text { Luminosité } \mathrm{L}^{*} \\
\text { Indice de rouge } \mathrm{a}^{*} \\
\text { Indice de jaune } \mathrm{b}^{*}\end{array}$ & $\begin{array}{c}4 \\
80,7 \pm 0,9 a \\
4,3 \pm 0,7 a \\
11,6 \pm 1,5 a\end{array}$ & $\begin{array}{c}4 \\
77,0 \pm 1,6 \mathrm{~b} \\
2,9 \pm 1,7 \mathrm{a} \\
25,1 \pm 4,4 \mathrm{~b}\end{array}$ & $\begin{array}{c}10 \\
71,5 \pm 2,1 \mathrm{c} \\
5,0 \pm 1,2 \mathrm{a} \\
14,3 \pm 2,9 \mathrm{a}\end{array}$ & $\begin{array}{c}4 \\
68,9 \pm 5,7 \mathrm{c} \\
7,0 \pm 3,5 \mathrm{ab} \\
15,0 \pm 1,4 \mathrm{c}\end{array}$ & $\begin{array}{c}5 \\
70,2 \pm 4,4 \mathrm{c} \\
8,7 \pm 1,7 \mathrm{~b} \\
14,3 \pm 2,3 \mathrm{a}\end{array}$ \\
\hline $\begin{array}{l}\text { Nombre d'échantillons } \\
\text { Teneur en caroténoïdes } \\
\text { (ug/g) } \\
\text { Teneur en bilirubine (ug/g) }\end{array}$ & $\begin{array}{c}4 \\
0,58 \pm 0,26 \mathrm{a} \\
0,20 \pm 0,08\end{array}$ & $\mid \begin{array}{c}4 \\
4,60 \pm 2,00 \mathrm{~b} \\
0,42 \pm 0,30\end{array}$ & & $\begin{array}{c}7 \\
0,64 \pm 0,53 \mathrm{a} \\
0,22 \pm 0,08\end{array}$ & \\
\hline
\end{tabular}

Test statistique non paramétrique de Mann et Whilney :

a, b, c : les valeurs homologues accompagnées de lettres différentes sont significativement différentes au seuil $P<0,05$.

Les maxima d'absorption: les gras blancs présentent 3 maxima d'absorption à 576,542 et $420 \mathrm{~nm}$ qui sont caractéristiques de la forme oxygénée des pigments héminiques dans le visible pour les deux premiers et dans le proche ultra violet pour le 3" (bande de Soret entre 415 et $425 \mathrm{~nm}$, Renerre 1988). Ces maxima se retrouvent dans tous les autres types de gras, soit aux mêmes longueurs d'onde (gras jaunes), soit légèrement décalés ou réduits à la forme d'épaulements dans les gras brun-rouge. Ces derniers ont été répartis en 3 types selon leur spectre :

* le type I (10 échantillons) qui présente 2 maxima à $555 \mathrm{~nm}$ (forme réduite des pigments héminiques) et entre 420 et $432 \mathrm{~nm}$ (bande de Soret) ainsi que 2 épaulements entre 570 et 625 $\mathrm{nm}$ puis entre 495 et $542 \mathrm{~nm}$. Ces 2 épaulements pourraient traduire un début d'oxydation des pigments caractérisés par leur couleur brune.

* le type II (5 échantillons) où l'on observe 3 pics dont la position est analogue à celle observée sur les échantillons blancs : $576 ; 545$ et la bande de Soret à $420 \mathrm{~nm}$. Les pigments héminiques y seraient sous leur forme oxygénée.

* le type III (4 échantillons) est intermédiaire entre les deux précédents. On y trouve 3 maxima d'absorption : à $576 \mathrm{~nm}$, entre 545-557 $\mathrm{nm}$ et entre $415-422 \mathrm{~nm}$ (bande de Soret) ainsi que 2 épaulements peu marqués (595 à 620 et 495 à $545 \mathrm{~nm}$ ). Les pigments héminiques seraient présents surtout sous les deux formes oxygénée et réduite.

Selon les conditions de conservation, une carcasse pourrait donc présenter un spectre de l'un des 3 types selon la proportion de pigments sous l'une ou l'autre forme. C'est bien ce que nous avons observé sur un échantillon qui est passé successivement de la forme réduite au moment de l'abattage (maxima vers $555 \mathrm{~nm}$ ) à la forme oxygénée après 12 heures de conservation à l'air puis de nouveau à la forme réduite après 3 jours de conservation sous vide.
Les gras jaunes se distinguent des autres par leur spectre d'absorption dans la zone 435-512 $\mathrm{nm}$. Deux des gras observés (1 et 2) présentent un maximum entre 460 et $480 \mathrm{~nm}$. les deux autres (3 et 4) un gros épaulement qui correspondent à la présence de pigments particuliers: caroténoïdes ou bilirubine. La méthode spectrocolorimétrique ne permet cependant pas d'identifier précisément le pigment en cause car les 2 types de composés ont un maximum d'absorption à des longueurs d'onde très voisines.

b / La proportion de lumière réfléchie: les spectres d'absorption se caractérisent non seulement par la position de leur maxima présentée plus haut mais aussi par leur amplitude. Les maxima d'absorption augmentent d'amplitude des gras blancs aux jaunes puis aux brunrouge. Il en résulte une réduction progressive de la quantité de lumière réfléchie. Celle-ci, mesurée à $700 \mathrm{~nm}$ passe de $75,3 \%$ pour les gras blancs à $70,9 \%$ pour les jaunes et $64,9 \%$ pour les brun-rouge sans différence sensible entre les 3 types de gras distingués dans ce cas.

\section{2 / Les indices colorimétriques du système CIELAB}

La luminosité des tissus adipeux $\left(\mathrm{L}^{*}\right)$ diminue significativement quand on passe des gras blancs $\left(L^{*}=80,7\right)$ aux jaunes $\left(L^{\star}=77,0\right)$ puis aux brun-rouge $\left(\mathrm{L}^{\star}=70,6\right)$; l'indice de rouge est maximum dans les gras brun-rouge ( $\mathrm{a}^{*}=$ $6,4)$ et l'indice de jaune dans les gras jaunes $\left(\mathrm{b}^{*}=25,1\right)$ (tableau 2).

Les indices $\mathrm{L}^{*}$, $\mathrm{a}^{*}$ et $\mathrm{b}^{*}$ sont stables d'un échantillon à l'autre dans le groupe des gras blancs. Les 4 échantillons jaunes sont beaucoup plus variables et se regroupent en 2 catégories : d'une part, les échantillons $n^{\prime \prime} 1$ et 2 qui présentent un indice de jaune très élevé et un indice de rouge proche de celui des gras blancs, d'autre part les échantillons $\mathrm{n}^{\text {" }} 3$ et 4 , dont l'indice de jaune est un peu moins élevé 
Tableau 3.Caractéristiques colorimétriques et teneurs en bilirubine et en pigments caroténoïdes des échantillons de tissu adipeux jaunes.

\begin{tabular}{|l|c|c|c|c|}
\hline \multicolumn{1}{|c|}{ Couleur } & Blanc & Jaune & \multicolumn{2}{c|}{ Brun-rouge } \\
\hline \multicolumn{1}{|c|}{ Type } & & & I & II \\
\hline Numéro de l'échantillon & 1 & 2 & 3 & 4 \\
Indices CIELAB & & & & 79,1 \\
Luminosité L* $_{\text {Indice de rouge a }}^{*}$ & 75,3 & 76,9 & 76,7 & 2,0 \\
Indice de jaune b* & 3,3 & 5,0 & 1,1 & 20,4 \\
Teneur en caroténö̈des $(\mu \mathrm{g} / \mathrm{g})$ & 30,1 & 27,5 & 22,7 & 5,14 \\
Teneur en bilirubine $(\mu \mathrm{g} / \mathrm{g})$ & 6,16 & 5,39 & 1,70 & 0,70 \\
\hline
\end{tabular}

Tableau AQuantité de caroténoïdes totaux présents dans le tissu adipeux d'ovins.

\begin{tabular}{|c|c|c|c|c|c|}
\hline \multirow[t]{2}{*}{ Auteurs } & \multirow[t]{2}{*}{ Nbre } & \multirow{2}{*}{$\begin{array}{l}\text { Animaux } \\
\text { Caractéristiques }\end{array}$} & \multicolumn{3}{|c|}{$\begin{array}{l}\text { Caroténoïdes totaux } \\
\text { tissu adipeux }(\mu \mathrm{g} / \mathrm{g})\end{array}$} \\
\hline & & & périrénal & mésentérique & sous-cutané \\
\hline Patterson 1965 & 2 & Animaux normaux & 0,30 à 0,50 & & \\
\hline Kirton et al 1975 & 1 & $\begin{array}{c}\text { Animal rejeté à l'exportation } \\
\text { pour graisse jaune }\end{array}$ & 2,93 & & \\
\hline Karijord 1978 & 3 & Animaux jaunes typiques & 2,20 & & \\
\hline Kirton et al 1975 & 1 & Mâle castré à couleur jaune & 3,96 & 8,64 & 3,82 \\
\hline Kirton et al 1975 & 3 & $\begin{array}{l}1 \text { brebis et } 2 \text { moutons rejetés à } \\
\text { l'exportation pour graisse jaune }\end{array}$ & 5,41 & 2,38 & 5,55 \\
\hline Rozier et al 1979 & 14 & Animaux à graisse jaune & $\begin{array}{c}5,36 \\
(0 \text { à } 21,00)\end{array}$ & & \\
\hline Karijord 1978 & 17 & Graisse blanche à jaune & 0,12 à 2,36 & & \\
\hline
\end{tabular}

et l'indice de rouge nettement inférieur à la normale (tableau 3). Les indices des échantillons brun-rouge sont également variables, en particulier l'indice de rouge qui passe de 5,0 dans les gras de type I à 7,0 et 8,7 dans ceux du type intermédiaire et du type 11 respectivement. Les variations des caractéristiques colorimétriques de la viande selon l'état d'oxydoréduction des pigments héminiques ont déjà été décrites.

probablement de

l'oxyhémoglobine résiduelle après saignée.

\section{3 / Concentration du tissu adipeux en pigments}

\section{a / Concentration en bilirubine et pigments caroténoïdes}

La concentration en pigments caroténoïdes des échantillons blancs varie de 0,3 à $0,8 \mu \mathrm{g} / \mathrm{g}$ (tableau 2) et leur teneur en bilirubine de 0,1 à $0,4 \mu \mathrm{g} / \mathrm{g}$, valeurs très voisines de celles observées sur les tissus adipeux brun-rouge.

Dans le cas des échantillons jaunes, les variations sont beaucoup plus importantes (tableau 3 ). Les échantillons $n^{\circ} 1$ et 2 ont des teneurs élevées en caroténoïdes, du même ordre que celles observées par différents auteurs sur des animaux jugés jaunes à très jaunes, c'est à dire de 4 à 10 fois plus importantes que la normale (tableau 4). Ces deux échantillons présentent de faibles teneurs en bilirubine, moins de $0,2 \mu \mathrm{g} / \mathrm{g}$ donc analogues à celles observées sur les gras blancs ou brun-rouge.

Les échantillons $n^{\circ} 3$ et $n^{\prime \prime} 4$ présentent de fortes teneurs en bilirubine, 3 à 4 fois supérieures à celles observées dans les gras blancs, brun-rouge ou les échantillons n"1 et 2 . Ceci est à rapprocher des faibles valeurs d'indice de rouge présentées par ces échantillons; on peut en effet supposer la présence de verdohémoglobine et/ou de biliverdine, pigments verts précédant la bilirubine dans le métabolisme de dégradation de l'hémoglobine, ou pouvant résulter de l'oxydation de la bilirubine pour le deuxième. L'échantillon 4 contient les 2 types de pigments : fortes teneurs en caroténoïdes et en bilirubine, malgré un indice de jaune relativement faible. On peut supposer qu'une coloration parasite liée à la peroxydation des lipides s'est développée au cours de l'analyse de cet échantillon comme le montre l'absorption caractéristique importante observée dans ce cas à $370 \mathrm{~nm}$ (Tappel 1955).

\section{b / Concentration en pigments héminiques}

Tous les échantillons observés contiennent des pigments héminiques mais sous des formes d'oxydoréduction qui peuvent être différentes, et à des concentrations croissantes quand on 
passe des gras blancs aux gras brun-rouge. La nature exacte des pigments héminiques dans ces différents tissus est en cours de détermination. La méthode mise en oeuvre nécessite en particulier la caractérisation de la protéine associée à l'hème. Les premières mesures réalisées sur un échantillon brun-rouge mettent en évidence la présence de protéines héminiques de poids moléculaires voisins de 68000,17000 et 12000 correspondant à l'hémoglobine pour la première, à un monomère de cette protéine (voire à la myoglobine) pour la seconde et à des cytochromes musculaires pour la troisième (Renerre 1977).

\section{3 / Les autres caractéristiques qui accompagnent les défauts de couleur : les défauts de fermeté}

Les tissus adipeux blancs et jaunes étaient fermes au toucher; en revanche, 18 des 19 tissus brun-rouges ont été jugés mous ou huileux.

La fermeté du tissu adipeux dépend essentiellement de sa teneur en lipides (donc de sa teneur en eau) et de sa composition en acides gras. Les tissus fermes, qui sont recherchés, se caractérisent par une forte teneur en lipides, une faible teneur en eau, par une proportion élevée d'acides gras saturés et une faible proportion d'acides gras insaturés, impairs ou ramifiés.

\section{1 / Teneur du tissu adipeux en lipides}

La teneur en lipides des échantillons blancs ou jaunes varie de 73 à $80 \%$ du poids frais pour les premiers et de 73 à $94 \%$ pour les seconds. Ces valeurs qui ne sont pas significativement différentes entre elles (tableau 5) le sont par contre de celles observées sur les gras brun-rouge ( 58 à $90 \%$ ). On observe en outre une plus forte variabilité dans les échantillons jaunes et brun-rouge que dans les blancs (coefficient de variation de 4,$2 ; 11,7$ et $15,8 \%$ respectivement pour les gras blancs, jaunes et brun-rouge).

\section{2 / Composition en acides gras}

Les tissus blancs ou jaunes ont des teneurs voisines pour les différents types d'acides gras (AG) des triglycérides : environ $40 \%$ d'AG aliphatiques (ou linéaires) saturés, $48 \%$ d'AG aliphatiques monoinsaturés ainsi que $5 \%$ d'AG impairs ou ramifiés et $5 \%$ de polyinsaturés (n-3) et (n-6) (tableau 5). Par contre les tissus brun-rouge présentent une composition en $\mathrm{AG}$ de triglycérides différente: significativement moins d'AG aliphatiques saturés $(32 \%)$ ou polyinsaturés (n-3: 1,5\%), légèrement plus d'AG aliphatiques monoinsaturés $(51,7 \%)$ ou polyinsaturés (n-6: 4,3\%) et près de deux fois plus d'AG impairs ou ramifiés $(9,8 \%)$.

Les phospholipides (AG des membranes cellulaires) ont des teneurs en $A G$ polyinsaturés en moyenne 3 à 5 fois plus élevées que celles observées dans les triglycérides. Cependant, les phospholipides des échantillons de gras jaune $\mathrm{n}^{\circ} 1$ et 2 (les plus riches en pigments caroténoïdes\} se différencient par leur teneur très élevée en AG saturés ( 71 et $76 \%$ ) et leur très faible teneur en $A G$ impairs et ramifiés ( 3 et $5 \%$ ), ainsi qu'en $\mathrm{AG}$ monoinsaturés aliphatiques (12 et $22 \%$ ) et polyinsaturés ( 3 et $6 \%$ ). Cette augmentation de la teneur en AG saturés semble liée à l'inhibition d'enzymes spécifiques (delta 9 - désaturases) chez les animaux abondamment approvisionnés en caroténoïdes (Alam et al 1984).

Par rapport à ceux des tissus blancs, les phospholipides des tissus brun-rouge présen-

Tableau 5. Teneur en lipides et composition en acides gras de tissus adipeux sous-cutanés prélevés sur des carcasses d'agneaux de boucherie de couleur blanche, jaune ou brun-rouge (moyenne et écart-

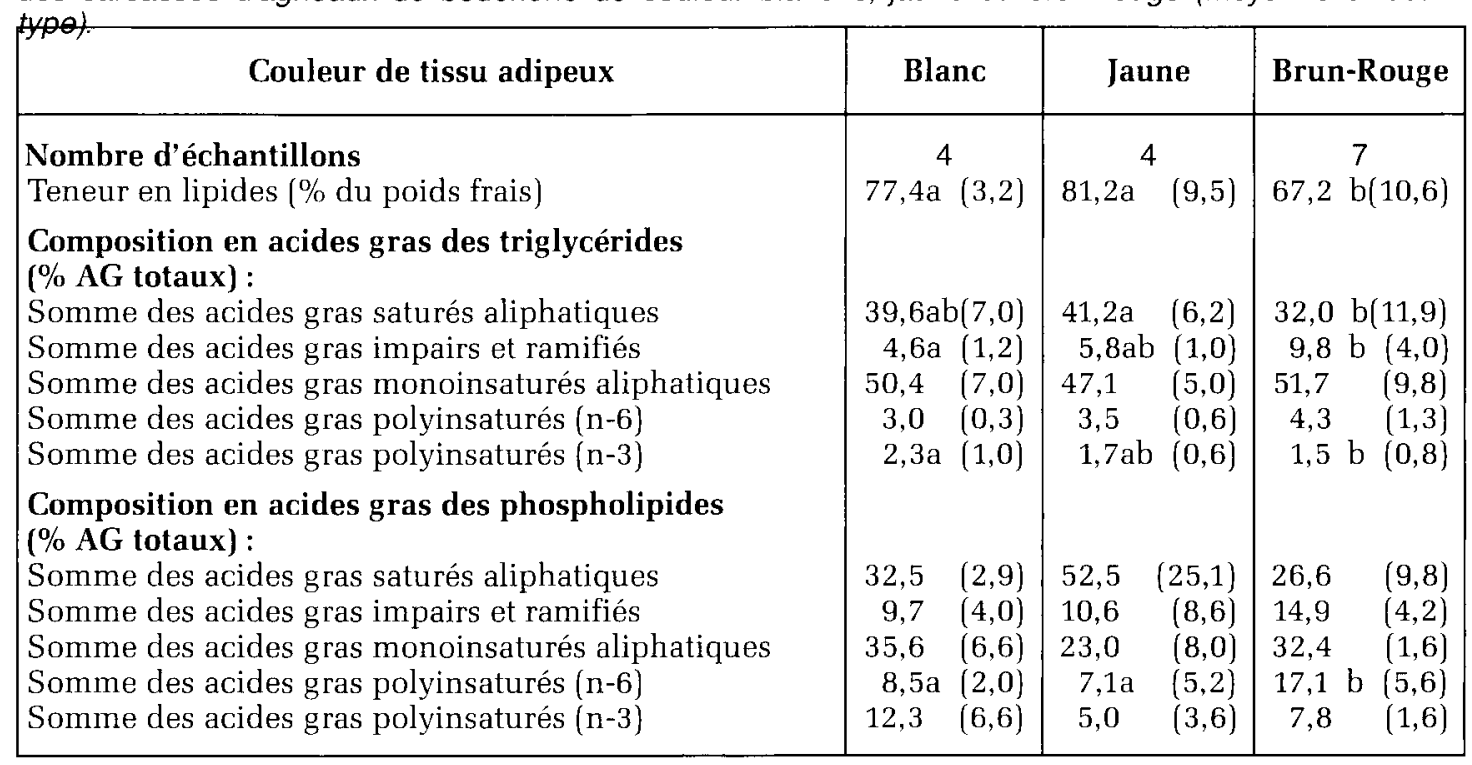

Test statistique de Mann et Whitney :

$a, b$ : les valeurs homologues accompagnées de lettres différentes sont significativement différentes au seuil Pro,05. 
tent une plus faible teneur en AG saturés, et des teneurs plus élevées en AG impairs et ramifiés ainsi qu'en AG polyinsaturés (n-6). Si les différences de teneur des phospholipides en AG monoinsaturés sont minimes entre les gras blancs et brun-rouge, ces derniers se caractérisent cependant par des proportions importantes d'isomères trans de l'acide oléique $(0,1$ à $4 \%$ ). Ces derniers, obtenus après hydrogénation incomplète des $A G$ insaturés dans le rumen (Tove et Matrone 1962), sont incorporés activement dans les phospholipides membranaires (Watkins 1989) et en particulier dans la membrane des globules rouges qu'ils fragilisent (Whale 1983 ; Tsao et Lands 1980) ce qui pourrait être à l'origine de la coloration brun-rouge de certains tissus adipeux.

Ces variations de composition en AG reflètent les conditions d'élevage des agneaux : les agneaux d'herbe présentent des phospholipides riches en $A G$ polyinsaturés (n-3), alors que l'utilisation de régimes plus concentrés entraîne une diminution des AG polyinsaturés ( $n-3)$ et une augmentation des AG polyinsaturés (n-6). Les modifications de composition du tissu adipeux en AG observées sur les échantillons brun-rouge sont typiques de celles rencontrées sur les agneaux de bergerie. Chez ces animaux, une partie importante des AG polyinsaturés $(n-6)$ apportés par les aliments concentrés peut échapper aux hydrogénations dans le rumen, et des proportions élevées d'AG impairs et ramifiés peuvent être synthétisés dans le tissu adipeux lorsque les capacités du foie à utiliser l'acide propionique pour la néoglucogénèse ont été débordées.

\section{4 / Origine des défauts de couleur du tissu adipeux}

\section{1 / Colorations jaunes}

\section{a / Accumulation excessive de pigments caroténoüdes}

L'identification de ces pigments d'origine alimentaire est délicate: faible concentration et difficultés de dosage. Les carotènes, qui colorent en jaune la graisse des bovins ne seraient pas présents chez les ovins où l'on trouve surtout des xanthophylles, dont la lutéine, qui est le principal responsable de la coloration des gras dans cette espèce. Les caroténoïdes ne sont synthétisés que par les plantes, les animaux ne peuvent, tout au plus, qu'en modifier légèrement la structure.

L'accumulation anormale de ces pigments dans les graisses a une origine mal connue car on connaît peu le métabolisme normal de leur dégradation dans l'organisme et encore moins bien ses troubles.

Si le métabolisme des caroténoïdes a fait l'objet d'études dans le cas des provitamines A (Goodman 1988), il n'en est pas de même pour ceux, dont la lutéine, qui ne sont pas des précurseurs de la vitamine A (Olson 1989). On a encore accordé très peu d'attention à leur devenir métabolique et à la régulation de celui-ci (Olson 1989), mais des études de la spécificité de la $\beta$-caroténoïd 15,15 '-dioxygenase qui scinde les provitamines $\mathrm{A}$ dans l'intestin, ont déjà révélé que cette enzyme était inefficace dans le clivage de la lutéine.

Compte tenu de l'état des connaissances, on en est encore réduit à ne formuler que des hypothèses sur les causes de l'accumulation anormale de ces pigments dans les graisses. Celle ci peut être due à :

- Un apport très important de caroténoïdes dans la ration pouvant dépasser les capacités de transformation de ces pigments par l'organisme (Krinsky et al 1989)

- Une altération du métabolisme de leur dégradation due à l'absence ou à la déficience d'enzymes intestinales ou hépatiques (Kirton 1980, Parker 1989, Rozier et al 1979, Olson 1989).

- Une origine génétique, démontrée récemment par Baker et al (1985) : leurs travaux semblent prouver que le caractère " graisse jaune » chez les ovins serait en partie dû à un gène majeur récessif, mais l'hypothèse d'un déterminisme polygénique plus complexe, n'est pas exclue. Le(s) gène(s) serai(en)t responsable(s) soit d'une déficience en enzymes intervenant dans l'oxydation des caroténoïdes (Baker et al 1985), soit d'une différence de spécificité de la $\beta$-carotenoïd-dioxygenase (Karijord 1978). L'héritabilité de ce caractère serait comprise entre 0,13 et 0,29 selon les races (Kirton et al 1975, Baker et al 1985)

Les seules méthodes efficaces pour réduire la fréquence des animaux porteurs sont l'élimination des reproducteurs ayant une descendance à problème et une gestion correcte de ces descendants (Karijord 1978, Baker et al 1985) ou plus rapide encore, leur sélection sur la base du taux de caroténoïdes sanguins (Baker et al 1985), puisque Karijord (1978) a montré qu'il existait une bonne relation entre la concentration en caroténoïdes du tissu adipeux et celle du plasma $(r=0,91)$.

\section{b / Accumulation excessive de bilirubine}

Les ictères ou jaunisses traduisent une accumulation excessive de bilirubine dans les tissus. Des analyses faites par Popoff et al (1980) sur des carcasses d'ovins à graisse jaune ont donné des teneurs de 2 à $45 \mathrm{mg}$ de bilirubine libre/ $\mathrm{kg}$ de tissu adipeux (12 $\mathrm{mg}$ en moyenne), alors que les teneurs obtenues sur des tissus normaux étaient inférieures à $5 \mathrm{mg} / \mathrm{kg}[0,7$ $\mathrm{mg} / \mathrm{kg}$ en moyenne). Les concentrations que nous obtenons: $0,2 \mathrm{mg} / \mathrm{kg}$ en moyenne dans les tissus adipeux normaux, $0,7 \mathrm{mg} / \mathrm{kg}$ dans les tissus adipeux ictériques analysés (échantillons jaunes n"3 et 4) sont beaucoup plus faibles, peut être à cause d'une pathologie moins importante mais aussi probablement du fait de méthodes d'extraction et de dosage différentes; il faut en particulier signaler que les méthodes utilisées par Popoff et al (1980) ne permettent pas de séparer les pigments caroténoïdes de la bilirubine.

La bilirubine dérive essentiellement du métabolisme de dégradation de l'hémoglobine. On distingue deux formes de bilirubine sanguine, dont la séparation est utile dans la détermination de l'origine de l'ictère 
- la bilirubine non conjuguée ou libre transportée dans le sang grâce à des liaisons relativement fortes avec l'albumine plasmatique.

- la bilirubine conjuguée au niveau du foie et excrétée par les canalicules biliaires: cotte forme ne se trouve normalement pas dans le sang.

Les ictères à bilirubine libre peuvent être dus à une surproduction de bilirubine à la suite d'une hémolyse importante pour cause infectieuse, parasitaire, microbienne (entérotoxémie), ou toxique (excès de cuivre dans la ration). Un dysfonctionnement du foie peut également entraîner des cas d'ictères à bilirubine libre (défaut de captation de la bilirubine par les cellules hépatiques, déficit de la glycuro-conjugaison) ou à bilirubine conjuguée (déficit du système enzymatique excréteur, obstacles à l'écoulement normal de la bile par suite de fibrose hépatique ou d'infestation parasitaire par la Grande Douve). Enfin des toxines diverses présentes dans les fourrages peuvent être à l'origine d'ictères hépatiques, sans que les mécanismes impliqués soient connus avec exactitude : c'est le cas de l'eczéma facial du mouton, bien connu en Nouvelle Zélande, et qui a été signalé récemment par Bonnefoi et al (1988) au Pays Basque français.

Compte tenu de cette diversité d'origines, il est indispensable d'établir un tableau clinique précis de l'ictère pour en déterminer la cause et y remédier.

\section{2 / Origine de la coloration brun-rouge}

Cette coloration pourrait avoir pour origines :

1 1") une variation dans la réflexion de la lumière selon le degré de fermeté du tissu adipeux (Girard, Aurousseau, communications personnelles).

2) l'accumulation excessive de pigments héminiques, du fait de la fragilisation de la membrane des globules rouges, provoquée par la peroxydation des phospholipides membranaires riches en acides gras insaturés (Buckley et al 1989, Faustman 1989) et/ou la présence de proportions importantes d'isomères trans de l'acide oléique dans ces phospholipides membranaires (Wahle 1983, Tsao et Lands 1980).

$3^{11)}$ le développement de produits colorés (lipopigments jaune-brun, ceroid et lipofuscine) lors de la peroxydation des graisses insaturées (Tappel 1955, Palmer et al 1986). Celle-ci aboutit à la formation de peroxydes et de composés divers tels que des aldéhydes, des cétones et des esters (Mayer et al 1953) qui brunissent (Tappel 195.5, Mayer et al 1953).

L'hypolhose de la peroxydation des graisses insalurés peut probablement être avancée dans la mesure où la coloration se développe et/ou s'accentue avec la durée de ressuyage de la carcasse. Les causes en seraient une concentration excessive d'acides gras insaturés dans le tissu adipeux (Tappel 1955, Palmer et al 1986), un excès d'apport de cuivre (Lovstad 1982) ou la stimulation de la peroxydation des acides gras insaturés par des pigments héminiques, que l'on retrouve à forte concentration dans les échantillons brun-rouge et qui seraient des catalyseurs de l'oxydation des graisses insatu- rées et de la formation des lipopigments (Tappel 1955 ; Buckley et al 1989). Une concentration insuffisante en antioxydants tels les phénols, les caroténoïdes, la vitamine $\mathrm{E}$, la cystine peut également être à l'origine de la coloration brun-rouge car ces substances diminuent l'oxydation et la peroxydation spontanée des acides gras insaturés. Certains auteurs remarquent cependant qu'il n'y a pas toujours parallélisme entre les variations de la peroxydation et celles de la concentration du tissu adipeux en lipopigments jaune-brun (Dam et al 1949 cités par Granados et al 1949), et que, d'autre part, l'effet des antioxydants diminue quand le degré d'insaturation des graisses augmente (Faustman 1989). Enfin, des essais de supplémentation en vitamine $\mathrm{E}$ d'agneaux de bergerie dont le régime contient moins d'antioxydants que l'herbe, n'ont pas réduit la coloration du gras sous cutané (Murphyy et L'Estrange 1977).

Le phénomène de peroxydation peut s'apprécier par différentes mesures (test TBA, indice de peroxyde), mais celles-ci posent des problèmes d'interprétation du fait qu'elles peuvent être entachées d'artefacts (décomposition des peroxydes, par exemple), et qu'elles ne sont pas directement en relation avec la quantité de composés colorés formés. C'est pourquoi ces mesures n'ont pas été effectuées sur les échantillons analysés ; la caractérisation et la quantification directes des lipopigments colorés nécessitent donc encore la mise au point de méthodes adaptées.

L'apparition de cette coloration brun-rouge et le manque de fermeté du tissu adipeux seraient donc deux phénomènes en grande partie associés. C'est pourquoi, la coloration brune concerne plus fréquemment les carcasses d'agneaux mâles d'une part (Paruelle et Pain 1982), et élevés en bergerie d'autre part (L'estrange et Mulvihill 1975). En effet, la teneur du tissu adipeux en AG insaturés et impairs est plus élevée chez les mâles que chez les femelles (Molénal et Thériez 1973) et les régimes riches en concentrés peuvent entraîner des modifications du métabolisme lipidique. En cas de consommation de quantités importantes d'aliments concentrés, en effet, une partie importante des AG polyinsaturés (n-6) peut échapper aux hydrogénations dans le rumen, et la production excessive d'acide propionique peut conduire à une augmentation des proportions d'AG impairs et ramifiés dans le tissu adipeux.

\section{Conclusion}

La méthode spectrocolorimétrique nous a permis d'avancer dans la description et la recherche de l'origine des défauts de coloration du tissu adipeux.

Les tissus adipeux blancs "de référence " correspondaient à des animaux produits exclusivement ou en grande partie à l'herbe. Nous y avons trouvé une faible quantité de pigments héminiques, probablement de l'oxyhémoglobine résiduelle dans les capillaires sanguins qui irriguent ce tissu. Les mesures réalisées à

\section{Le tissu adipeux brun-rouge contient probablement une quantité importante de pigments héminiques et des composés colorés formés lors de la peroxydation des acides gras insaturés.}


l'abattoir et en laboratoire ont montré qu'ils ne présentaient pas de défauts de fermeté.

Les tissus adipeux jaunes, qui ne présentaient pas non plus de défauts de fermeté et dont la composition en acides gras était voisine de celle des gras blancs, étaient colorés par des pigments caroténoïdes pour deux d'entre eux, par de la bilirubine pour le troisième et par les deux types de pigments pour le dernier. Le manque de connaissances actuelles quant au métabolisme de dégradation de la lutéine, qui est le principal pigment impliqué, ne permet pas de connaître précisément l'origine de l'excès de pigments caroténoïdes, mais on sait qu'elle comporte une forte composante génétique. Les causes de l'accumulation excessive de bilirubine sont par contre beaucoup mieux connues, mais elles sont très diverses.

Les tissus adipeux brun-rouge se caractérisaient le plus souvent par des défauts de fermeté associés aux défauts de couleur. Les animaux correspondants avaient généralement été élevés ou finis avec des rations comportant des proportions non négligeables de concentré. Ils présentaient, par rapport aux tissus adipeux blancs une moindre teneur en lipides, ainsi qu'une composition en acides gras des triglycérides et des phospholipides modifiée : en particulier moindre teneur en acides gras saturés ot plus forte teneur en acides gras impairs et ramifiés. Les différentes analyses effectuées onl révélé la présence de pigments héminiques à une concentration nettement plus élevée que dans les tissus adipeux blancs, ceci pouvant résulter de la fragilisation de la membrane des globules rouges compte tenu des caractéristiques de composition des phospholipides en acides gras. Le défaut de couleur peut être attribué 1) à une variation dans la réflexion de la lumière selon le degré de fermeté du tissu adipeux, 2) à l'accumulation excessive de pigments héminiques, 3) aux produits de la peroxydation des AG insaturés, elle même stimulée par la présence de ces pigments. Il reste à tester la validité de ces hypothèses en caractérisant la nature exacte des pigments impliqués.

Dans l'attente de techniques permettant d'annuler ces défauts, ou de la sélection d'animaux ne les présentant pas - on sait en effet que la fermeté du tissu adipeux comporte une composante génétique non négligeable (Bouix, communication personnelle)-, on ne peut que rappeler les précautions à prendre pendant l'engraissement en bergerie pour les limiter :

- éviter l'apport excessif dans la ration de matières grasses riches en acides gras insaturés.

- utiliser du bon foin lors de la finition des agneaux (au moins $20 \%$ de la ration), et préférer les céréales entières aux céréales broyées dans les régimes fermiers.

- éventuellement limiter l'apport d'aliment concentré pendant la période d'engraissement ou tout au moins pendant la phase de finition, et ainsi augmenter la part de fourrages dans le régime.

Ces précautions permettent de diminuer la vitesse de transit des aliments, de maintenir une sécrétion salivaire élevée et d'augmenter la capacité de la flore du rumen à hydrogéner les graisses insaturées. Elles évitent en effet les chutes trop importantes du pH qui entraînent une modification de la flore, une réduction de la production d'hydrogène et une trop forte proportion d'AG insaturés (Tove et Matrone 1962). Elles réduisent également la production d'acide propionique dont l'excès est responsable de la production d'acides gras impairs ou ramifiés.

L'intérêt de ces recommandations, présentées dès 1976 aux Journées de la Recherche Ovine et Caprine par Thériez, Van Quackebeke et Cazes, a été confirmé par Paruelle et Pain (1982) qui ont également observé une amélioration de la fermeté du gras sous cutané et une réduction de la fréquence des défauts de couleur en remplaçant la paille par du foin ou en limitant l'apport d'aliments concentrés à des agneaux de bergerie.

\section{Remerciements}

Nous remercions les différents organismes de Développement cités pour leur collaboration. Cette étude a pu être réalisée grâce au concours financier du FIDAR (Fonds Interministériel de Développement et d'Aménagement Rural).

\section{Références bibliographiques}

U'Ku sSlill B., 1986. Influence de l'alimentation et des facteurs d'élevage sur l'état d'engraissement et la qualité des carcasses chez les ovins. 11"s Journées Rech. Ovine et Caprine, 1-16.

BAKER R.L., STEINE T., VABENO A.W., BREINES D., 1985. The inheritance and incidence of yellow fat in norwegian sheep. Acta Agric. Scand., 35, 389-397.

BONNFFOI M., BEZILLE P., ARVAY O., BRAUN J.P, LE BARS J. 1988a. Impact économique de l'eczéma facial au Pays Basque: étude préliminaire. Revue Méd. Vét., 5, 489-492.

BUCKLEY D.I., GRAY J.I., ASGHAR A. PRICE J.F CRACKEL R.L., BOOREN A.M. PEARSON A.M., MIL LER F.R., 1989. Effects of dietary antioxidants and oxidized oil on membranal lipid stability and pork product quality. J. of Food Science, 54 (5), 1193-1197.

FAUSTMAN C., CASSENS R.G., SCHAEFER D.M., BUEGE D.R., WILLIAMS S.N., SCHELLER K.K., 1989. Improvement of pigment and lipid stability in holstein steer beef by dietary supplementation with vitamin $E$. J. Food Sci., 54 (4), 858-862.

ERANCIS FJ, CLYDESDALE FM, 1975, Food calorimetry: theory and applications. AVI Publishing comp. Westport, Connecticut.

GIRARD J.P., 1984. Les composantes de la qualité de la viande, du tissu adipeux, et des produits carnés. BulL. Tech. C.R.Z.V. Theix, INRA, 56, 59-67.

GOODMAN D.S., 1988. Vitamin A metabolism and the liver. In : The Liver: Biology and Pathobiology, Second Edition. Ed. by Arias I.M., Jakoby W.B., Popper H., Schachter D., Shafritz D.A., 467-474.

KARIJORD O., 1978. Correlation between the content of carotenoids in depot fat and in plasma of sheep. Acta Agricultural Scandinavica, 28, 355-359.

KIRTON A.H., 1980. Yellow fat in sheep. N.Z. Journal of Agriculture, 131 (6), 15-16.

KIRTON A.H., CRANE Brenda, PATERSON D.J., CLARE N.T., 1975. Yellow fat in lambs caused by carotenoid pigmentation. N.Z. Journal of Agricultural Research, 18 $267-272$.

KRINSKY N.I., 1989. Carotenoids and cancer in animal models. The Journal of Nutrition, 119 (1), 123-126. 
LESTRANGE J.L., MULVIHILL T.A., 1975. A survey of fat characteristics of lamb with particular reference to the soft fat condition in intensively fed lambs. J. Agric. Sci. Camb., 84, 281-290.

MOLENAT G., THERIEZ M., 1973. Influence du mode d'élevage sur la qualité de carcasse de l'agneau de bergerie. Ann. Zonter.h.. 22, 279-293.

OLSON J.A., 1989. Provitamin A function of carotenoids: the conversion of $\beta$-carotene into vitamin $A$. The Journal of Nutrition, 119 (1), 105-108.

PALMER D.N., HUSBANDS D.R., WINTER P.J, BLUNT J.W., JOLLY R.D., 1986. Ceroid lipofuscinosis in sheep. I Bis (Monoacylglycero) phosphate, dolichol, ubiquinone, phospholipids, fatty acids and fluorescence in liver lipopigment lipids. The J. of Biological Chemistry, 261 (4), 1766-1772.

PARKER R.S., 1989. Carotenoids in human blood and tissues. The Journal of Nutrition, 119 (1), 101-104.

PARUELLE J.L., PAIN J.M., 1982. Alimentation et qualité des graisses de dépôt des carcasses d'agneaux de bergerie. Bull. Tech. de l'ucaab, 1, 3-10.

POPOFF M., MENEROUD M., PETIT A., TASSIN P. BELINSKY 'C., LALOU-KERALY F.X., CHRISTODOU' LAKIS F., 1980. Carcasses d'ovins à coloration jaune Etudes bactériologiques et chimiques. Bull. Soc. Vét Prat de France, T. 64, N" 2. 155-171.

RENERRE M., 1977. Purification rapide et sous forme non oxydée de la myoglobine des viandes de bovins, ovins et porçins. Ann. Technol. Agric., 26 (4), 419-433.

RENERRE M., 1984. Variabilité entre muscles et entre animaux de la stabilité de la couleur des viandes bovines. Sci. Aliments, 4, 567-584.
RENERRE M., 1988. Quelles recommandations pour mesurer la couleur de la viande au laboratoire? I.A.A, 530-533.

ROZIER J., FERRANDO R., CARLIER V., FOURLON Claudine, 1979. Nature des graisses jaunes chez le mouton. Bull. Acad. Vét. de France, 1979, 52, 545-552.

SMITH G.C., CARPENTER Z.I., 1970. Lamb carcass quality. III Chemical, Physical and Histological measurements. J. Anim. Sci., 31, 697-706.

TAPPEL A.L., 1955. Studies of the mechanism of vitamin $\mathrm{E}$ action. III - In vitro copolymerization of oxidized fats with protein. Archives of Biochem. and Biophys., 54, 266-280.

THERIEZ M., VAN QUACKEBEKE E., CAZFS J.P., 1976. Influence de l'alimentation sur la croissance, l'état d'engraissement et la qualité des carcasses. 2èmes Journées de la Recherche Ovine et Caprine, 79-109.

TOVE S.B., MATRONE F. G.J., 1962. Effect of purified diets on the fatty acid composition of sheep tallow. J. Nutr., 76, 271-277.

TSAO Y.K., LANDS W.E.M., 1980. Cell growth with trans fatty acids is affected by Adenosine 3'-5'-Monophosphate and membrane fluidity. Science, 207, 777-779.

WAHLE K.J., 1983. Fatty acid modification and membrane lipids. Proc. Nutr. Soc., 42, 273-287.

WATKINS B.A., 1989. Influences of biotin deficiency and dietary trans-fatty acids on tissue lipids in chickens. Brit. J. Nutr., 61[1], 99-111.

\section{Summary}

\section{Discoloration of sheep carcass subcutaneous fat}

Color and firmness characteristics of 27 samples of lamb carcass subcutaneous fat $(4$ white, 4 yellow, 19 brown-red) are described using different methods (including spectrocolorimetry). For some samples, data on lamb fattening conditions were available.

White adipose tissues, considered as normal, were firm ; a low concentration in heme pigments was observed (probably residual oxyhemoglobin after slaughter). These samples came from grazing lambs.

Yellow adipose tissues were pigmented with carotenoïds and/or bilirubin. Their firmness was rather similar to white adipose tissues. Reasons for yellow discoloration are presented.

Most brown-red adipose tissues came from animals that were fattened with concentrates. These tissues were soft because of a lower lipid proportion and a change in fatty acid composition. Brown-red discoloration has been explained by 1) an effect of softness on light reflectance, 2 ) an excess in heme pigment concentration, 3) peroxydation of insaturated fatty acids.

PRACHE Sophie, AUROUSSEAU B., THERIEZ M., RENERRE M., 1990. Les défauts de couleur du tissu adipeux sous cutané des carcasses d'ovins. INRA Prod. Anim., 3, [4], 275-285. 